\title{
TCMS inhibits ATP synthesis in mitochondria: A systematic analysis of the inhibitory mechanism is
}

\author{
Marcantonio Bragadin ${ }^{\mathrm{a}, *}$, Alessandra Iero ${ }^{\mathrm{b}}$, Francesca Cima ${ }^{\mathrm{c}}$, \\ Loriano Ballarin $^{\mathrm{c}}$, Sabrina Manente ${ }^{\mathrm{a}}$ \\ a Environmental Sciences Department, Ca' Foscari University of Venice, Dorsoduro 2137, 30123 Venice, Italy \\ ${ }^{\mathrm{b}}$ Institute of Applied Ecology, Health Science and Design, University of Canberra, Bruce, ACT, Australia \\ ${ }^{\mathrm{c}}$ Biology Department, University of Padova, Viale G. Colombo 3 30121, Padova, Italy
}

Received 24 November 2006; accepted 16 April 2007

Available online 27 April 2007

\begin{abstract}
The interactions of the antifouling compound TCMS (2,3,5,6-tetrachloro-4-methylsulphonyl pyridine) with rat liver mitochondria have been investigated. The results indicate that the compound inhibits ATP synthesis. Further investigations regarding the ATP synthesis mechanism suggest that TCMS inhibits succinic dehydrogenase of the mitochondrial respiratory chain. As the respiratory chain is similar in all living organisms, it can be concluded that the toxic effect of TCMS most likely depend on the different bioavailability of the compound and on the different importance of mitochondria in the ATP production in the animal species.
\end{abstract}

(C) 2007 Elsevier Ltd. All rights reserved.

Keywords: Mitochondria; TCMS; ATP synthesis

\section{Introduction}

Many chemical compounds have been utilised as antifouling compounds as a means of protecting the bottom of boats from biomass growth.

The most famous and most widely utilised antifouling compound was tributyltin (TBT), but it is now banned from use. In fact, the ideal antifouling compound should be selective towards the biological species responsible for the growth of the biomass on the boats, but at the same time/on the other hand harmless towards the non-target biological species.

TBT does not satisfy this condition: it is highly efficient as an antifouling compound, but is extremely toxic for many living organisms. Furthermore, it accumulates in the food chain and is persistent in the environment. For

\footnotetext{
The work was carried out it the Environmental Sciences Department of Ca' Foscari University of Venice, Dorsoduro 2137, 30123 Venice, Italy.

* Corresponding author. Tel.: +39 041234 8507; fax: +39041234 8953.

E-mail address: bragadin@unive.it (M. Bragadin).
}

these reasons, many alternative antifouling compounds have been proposed.

TCMS (2,3,5,6-tetrachloro-4-methylsulphonyl pyridine), which was used in both the textile and leather industries, is one of the more recent antifouling compounds on the market. The toxicity of TCMS and other antifouling compounds towards living organisms has already been evidenced (Huh et al., 2001; Voulvoilis et al., 2001; Kostantinou and Albanis, 2004) and consequently, substantiated "in vitro" studies (Karlsson and Eklund, 2004; Sabev et al., 2004) in cells have subsequently been performed. The aim of this work is to study the interaction of TCMS with mitochondria, since, like TBT, mitochondria are a preferred target. We have verified that TCMS actually inhibits ATP synthesis. Consequently, we have systematically analysed each step involved in the ATP synthesis in order to individuate the molecular mechanism responsible for the ATP synthesis inhibition.

The step which is inhibited by the lowest dose of TCMS is succinic dehydrogenase in the mitochondrial respiratory chain $(\mathrm{RC})$. 
This suggests that, as the $\mathrm{RC}$ is similar in all living organisms, where the mitochondria are the preferential biological target, the sensitivity, towards TCMS should be similar.

\section{Materials and methods}

\subsection{Mitochondrial preparation}

Mitochondria were prepared from livers of fasted albino Wistar rats, weighing about $300 \mathrm{~g}$ (Bragadin et al., 1980). The mitochondrial protein concentration was determined using the Lowry procedure (Lowry et al., 1951).

\subsection{Oxygen consumption measurement (respiratory rate experiments)}

The mitochondrial oxygen consumption was measured using a Clark oxygen electrode (Yellow Springs Instruments, Yellow Springs, OH, USA) fitted in a thermostatcontrolled, closed chamber, equipped with a magnetic stirrer. The reaction medium $(2 \mathrm{ml})$ was kept thermostated at $20^{\circ} \mathrm{C}$ throughout all the experiments (standard in vitro conditions) (Azzone et al., 1979).

The oxidizable substrates utilised were: succinate $(1 \mathrm{mM})$, glutamate/malate $(2 \mathrm{mM})$ and ascorbate $(1 \mathrm{mM}) /$ $N, N, N^{\prime}, N^{\prime}$-tetramethyl- $p$-phenylenediamine (TMPD) (400 $\mu \mathrm{M})$.

Medium composition (experiments of Fig. 3): $0.25 \mathrm{M}$ sucrose, $10 \mathrm{mM}$ Hepes-Mops $\mathrm{pH}$ 7.4, $1 \mathrm{mM}$ EGTA, $5 \mathrm{mM} \mathrm{MgCl} 2,0.5 \mathrm{mM}$ sodium phosphate (Pi).

\subsection{Swelling experiments}

The swelling experiments were performed in a Jenway 6400 (Felsted, Essex, UK) spectrophotometer, equipped with a stirring apparatus, following the absorbance decrease at $540 \mathrm{~nm}$. After addition of the mitochondria to the resuspending medium $(2.5 \mathrm{ml})$, the instrument was adjusted to zero absorbance (Bernardi, 1999). Medium composition (experiments of Fig. 6): $0.25 \mathrm{M}$ sucrose, $10 \mathrm{mM}$ Tris-Hepes $\mathrm{pH} 7.4,1 \mathrm{mM}$ EGTA, $5 \mathrm{mM} \mathrm{MgCl}_{2}$, $0.5 \mathrm{mM}$ sodium phosphate $(\mathrm{Pi}), 1 \mathrm{mM}$ succinate.

\subsection{ATP synthesis/hydrolysis experiments}

The ATP synthesis/hydrolysis experiments were performed in a low buffered medium, using a $\mathrm{pH}$ meter 84 (Radiometer, Copenhagen, DK) monitoring, under stirring conditions at room temperature, the $\mathrm{pH}$ changes which accompany the reaction:

$\mathrm{ADP}+\mathrm{Pi}+\mathrm{H}^{+} \leftrightarrow \mathrm{ATP}$

(Nishimura et al., 1962).

Composition of medium used for ATP synthesis analysis (experiments of Fig. 2): $0.25 \mathrm{M}$ sucrose, $0.1 \mathrm{mM}$ HepesMops pH 7.4, $1 \mathrm{mM}$ EGTA, $0.5 \mathrm{mM}$ ADP, $5 \mathrm{mM} \mathrm{MgCl}_{2}$, $0.5 \mathrm{mM}$ sodium phosphate $(\mathrm{Pi})$.

Composition of medium used for ATP hydrolysis measurement (experiments of Figs. 4 and 5): $0.25 \mathrm{M}$ sucrose, $0.1 \mathrm{mM}$ Hepes-Mops pH 7.4, $1 \mathrm{mM}$ EGTA, $5 \mathrm{mM} \mathrm{MgCl} 2$, $0.5 \mathrm{mM}$ ATP, $50 \mathrm{nM}$ FCCP.

\subsection{Reagents}

FCCP (carbonylcyanide- $p$-trifluoromethoxyphenyl hydrazone), sodium glutamate, sodium malate, sodium hydrogen phosphate (Pi), oligomycin, TMPD and cyclosporine, were all obtained from SIGMA, Milan, Italy. The TCMS (Densil S 100) (purity 97\% assayed by HPLC (Thomas, 1998)). was supplied by Eigenmann \& Veronelli, Rho, MI, Italy.

All other reagents were of analytical grade.

\section{Results and discussion}

In mitochondria, the substrates arising from the Krebs cycle are oxidized by molecular oxygen producing ATP. The substrates oxidation is mediated by the mitochondrial respiratory chain (RC) (Fig. 1). The chemiosmotic hypothesis which explains the mechanism which drives the ATP synthesis (Mitchell, 1969), asserts that the electron flow in the $\mathrm{RC}$ is accompanied by a (stoichiometric) proton extrusion. Since the mitochondrial membrane is not permeable to the protons, their extrusion gives rise to a $\Delta \mathrm{pH}$ and a $\Delta \psi$, the sum of which constitutes the proton motive force (p.m.f.)

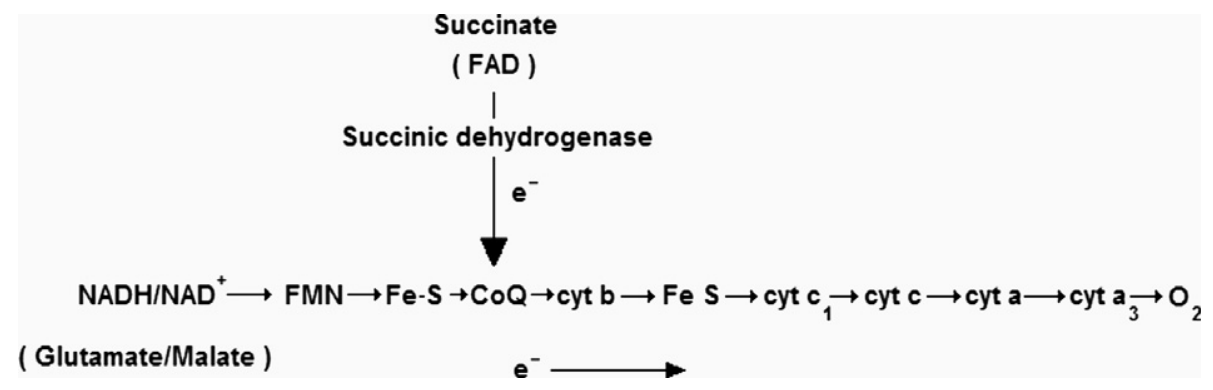

Fig. 1. The respiratory chain in mitochondria. In mitochondria, the electron flow from the reducing substrates to the oxygen, occurs by means of a sequence of redox couples, the mitochondrial respiratory chain (RC). The electron flow in the RC is coupled to the ATP synthesis. 
p.m.f. $=\Delta \mathrm{pH}+\Delta \psi$

which is the energy utilised to synthetize ATP from $\mathrm{ADP}+\mathrm{Pi}$

$\mathrm{ADP}+\mathrm{Pi} \underset{\text { ATPase }}{\stackrel{\text { energy }}{\longrightarrow}} \mathrm{ATP}$

As a consequence, if the ATP synthesis is inhibited, this may be due to:

- an inhibition of the RC,

- an inhibition of the ATPase (an enzyme which catalyzes ATP synthesis),

- an enhancement of the membrane permeability.

In turn, an enhancement of the membrane permeability can be due to:

(a) an enhancement of the proton permeability created by the protonophores (uncoupling effect);

(b) a detergent effect, which is an enhancement of the membrane permeability of all solutes (including protons);

(c) the opening of a membrane pore, which causes the passage of large-size solutes.

Taking into account the fact that TCMS actually inhibits ATP synthesis, as depicted in Fig. 2 (explanation further in text), each step in the process was separately analysed, in order to individuate which is responsible for the ATP synthesis inhibition.

\subsection{The respiratory chain $(R C)$}

It is obvious that an inhibition of the $\mathrm{RC}$ gives rise to an equivalent inhibition of the ATP synthesis. Therefore, this step was investigated, using glutammate/malate and succinate as oxidizable substrates which allows to analyse the functioning of the whole RC or only two oxidative phosphorylation sites, as illustrated by Fig. 1. Since the substrate oxidation causes the reduction of the oxygen present in the solution, the respiratory rate can be estimated from the rate of oxygen concentration decrease.

In Fig. 3a, a representative trace of succinate oxidation is reported. TCMS causes a decrease of the respiratory rate and this inhibitory effect is time dependent. For this reason, the respiratory rate inhibition was measured after a period of $10 \mathrm{~min}$ pre-incubation of TCMS with mitochondria. Then succinate was added and the respiration was stimulated by the addition of ADP (or of an uncoupler, such as FCCP). The stimulation caused by the uncoupler or by ADP $(+\mathrm{Pi})$ induces the maximal respiratory rate (Fig. 3a).

The respiratory rate values are reported in Fig. 3b. The figure shows that an inhibition of the RC occurs when the substrate is succinate, whereas, when the substrate is glutamate/malate, no respiratory rate inhibition occurs (not shown). These results suggest (Bragadin and Dell'Antone, 1994; Bragadin et al., 2005, 2006) hindering of the succinic dehydrogenase activity (Fig. 1).
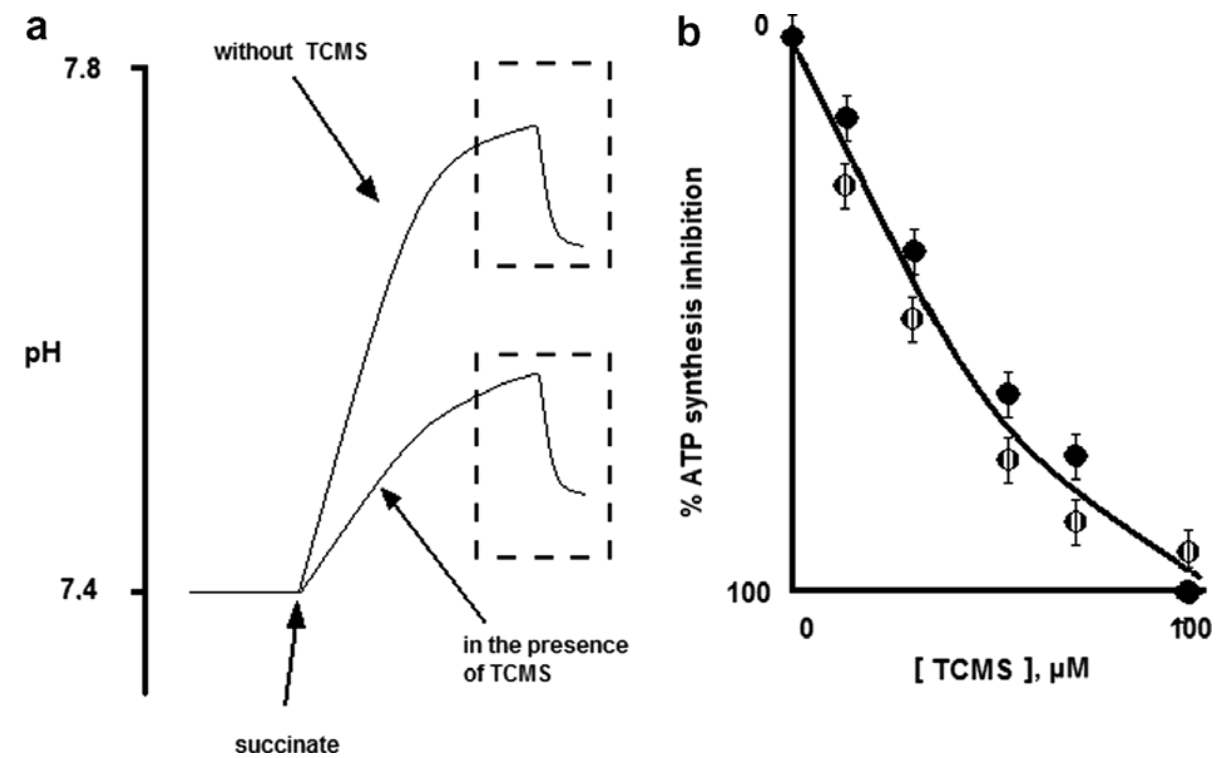

Fig. 2. The ATP synthesis is inhibited by TCMS. After the addition of the mitochondria (final concentration $1 \mathrm{mg}$ protein/ml), the medium was incubated for $10 \mathrm{~min}$ at $20^{\circ} \mathrm{C}$ in the presence of varying amounts of TCMS, before the addition of $1 \mathrm{mM}$ succinate. The pH changes were monitored following the procedure indicated in the Methods. In (a) a typical experiment. The graph in (b) reports the initial alkalinization rates after the addition of succinate in the absence $(\bigcirc)$ and in the presence of $1 \gamma$ cyclosporine $(\bigcirc)$ in the medium. Each experiment is the mean value of four replicative experiments. The sketched square in (a) evidences and acidification which occurs after ADP consumption. This behaviour suggests that the ATPase is not inhibited by TCMS (as evidenced in the experiments in Fig. 4 shown). 

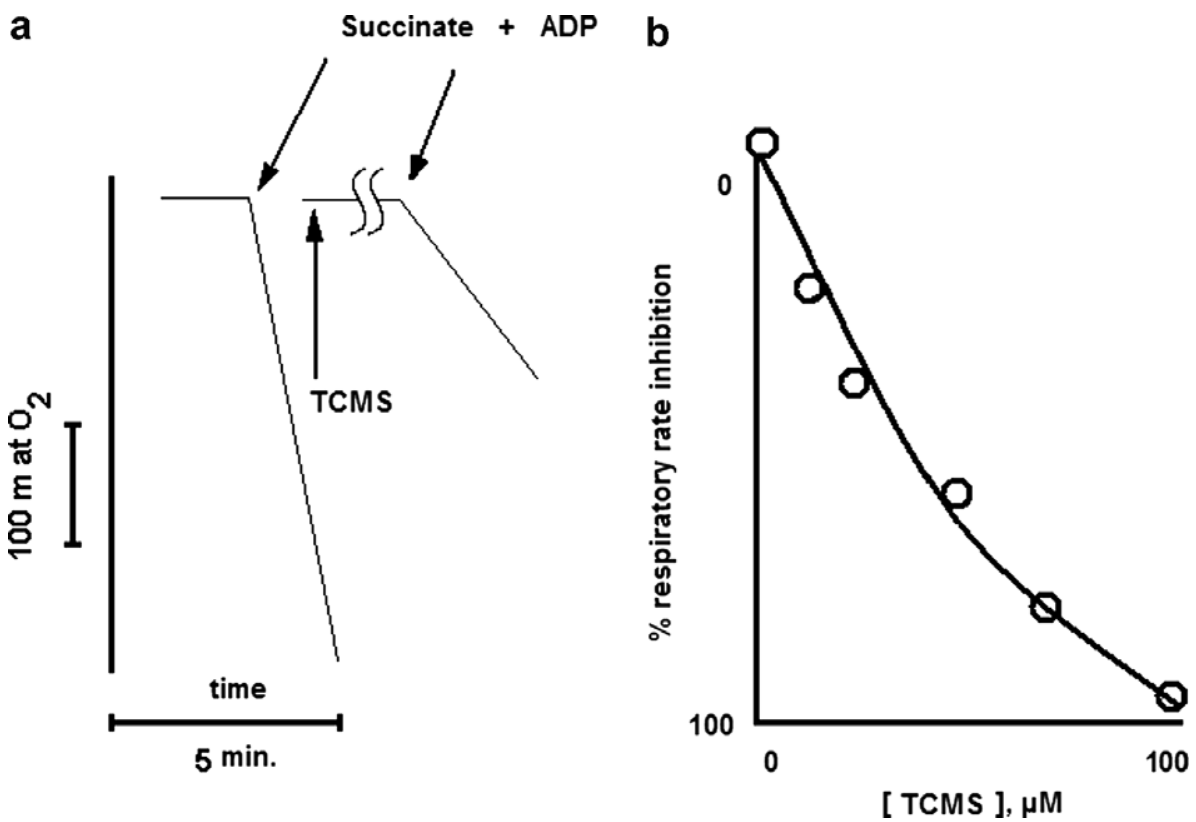

Fig. 3. TCMS inhibits the mitochondrial respiratory rate when succinate is the reducing substrate. After the addition of the mitochondria (final concentration $1 \mathrm{mg} / \mathrm{ml})$ and TCMS to the medium, the medium was incubated for 10 min before the addition of succinate (1 mM) and ADP $(0.5 \mathrm{mM})$ (or of succinate plus $50 \mathrm{nM}$ FCCP). Figure (a) reports a typical experiment in the absence and in the presence of TCMS. The graph in (b) reports the respiratory rate and its inhibition by TCMS. No inhibition was observed when using glutamate/malate $(2 \mathrm{mM})$ as a reducing substrate.

\subsection{The ATPase}

ATPase is the enzyme which catalyzes either ATP synthesis or ATP hydrolysis. In order to assess whether TCMS inhibits the ATP hydrolysis, the acidification process (which accompanies ATP hydrolysis) in the presence of TCMS, was measured. The ATP hydrolysis was measured in the presence of an uncoupler (FCCP) which permeates the membrane to the protons (see below) and, consequently, maximally enhances the rate of ATP hydrolysis. Under these conditions, the addition of oligomycin, which is a potent ATPase inhibitor, induces a complete inhibition of the acidification of the medium, while the addition of TCMS up to $400 \mu \mathrm{M}$ does not inhibit the medium acidification (Fig. 4). The experiments reported in Fig. 2 (see broken-line square in Fig. 2a), are consistent with this observation. Therefore we concluded that TCMS is not an inhibitor of ATPase and that the ATP synthesis inhibition cannot be ascribed to this effect.
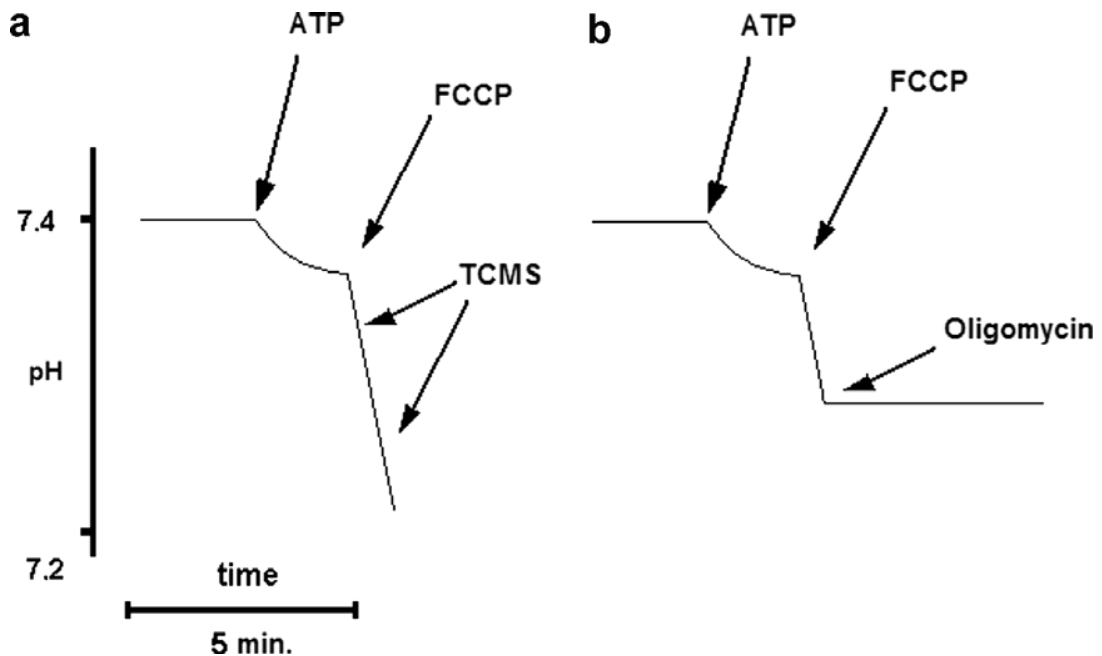

Fig. 4. TCMS does not inhibit ATPase. Since ATPase is the enzyme which catalyzes the ATP synthesis or hydrolysis, as is shown in the experiments in Fig. 2, the acidification rate was measured in a low buffered medium. In (a), the addition of increasing amounts of TCMS did not induce any inhibition in the acidification rate. For comparison purposes, in (b), the effect of $1 \gamma$ oligomycin, which is a potent ATPase inhibitor, was shown. 


\subsection{Enhancement of membrane permeability}

(a) Uncoupling effect. Proton conductivity enhancement is caused by a group of chemical compounds called uncouplers. The first uncoupler studied was DNP (2,4-dinitrophenol) whose uncoupling mechanism was explained by means of the chemiosmotic hypothesis (Mitchell, 1969).

In energised mitochondria, the internal alkaline $\mathrm{pH}$ drives the uptake of DNP as undissociated weak permeant acid. Once inside, the DNP is extruded as a phenate anion and the extrusion occurs as a consequence of a negative inside potential. The resulting whole mechanism is cyclic and, at any cycle, the net balance is a proton transport into the matrix. As TCMS is not a weak acid, it cannot act as a classical uncoupler. Nonetheless, we eliminated such a possibility by means of the following experiment: the mitochondria were energized with ATP, in a low buffered medium; the energization was followed by a slowing down to a steady state, in which the ATP hydrolysis rate was very low as shown in Fig. 4. This is due to the fact that the ATP-induced membrane energization is a reversible process which can be described as the sum of $\Delta \mathrm{pH}$ and $\Delta \psi$. Both the $\Delta \mathrm{pH}$ and $\Delta \psi$ are forces which oppose further ATP hydrolysis. The addition of an uncoupler (either FCCP or DNP) which enhances the proton permeability and consequently collapses the $\Delta \mathrm{pH}$ and $\Delta \psi$, induces a rapid ATP hydrolysis (Fig. 4a).

No ATP hydrolysis stimulation occurs (Fig. 5) if TCMS is added instead of FCCP.

(b) Detergent effect. The experiments of Fig. 5 clear indicate that TCMS is not an uncoupler (as expected, since TCMS is not a weak acid) and a detergent, since

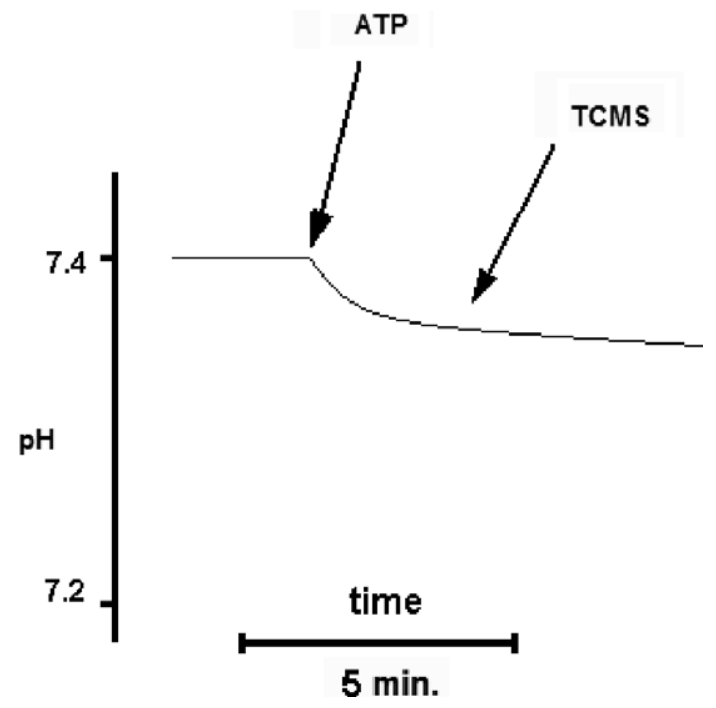

Fig. 5. TCMS is neither a protonophore nor a detergent. The experimental conditions were the same as those used in Fig. 4. TCMS was added instead of FCCP, but, at every level of TCMS concentration, no ATP hydrolysis stimulation occurred. This allowed us to conclude that TCMS is neither a protonophore nor a detergent compound. See for comparison the effect of the uncoupler FCCP which is added in the same conditions in Fig. 4.

the detergents enhance the membrane permeability to all solutes, the protons enclosed.

The experiments in Fig. 4 were made possible by the fact that TCMS does not inhibit the ATPase (Fig. 4).

(c) Opening of a membrane pore. Some chemical compounds induce the opening of a membrane permeability pore (MPT) (Bernardi, 1999) which allows for the passage of large molecule solutes, such as sucrose.

The entrance of sucrose induces the entry of water and consequently mitochondrial swelling as result of a
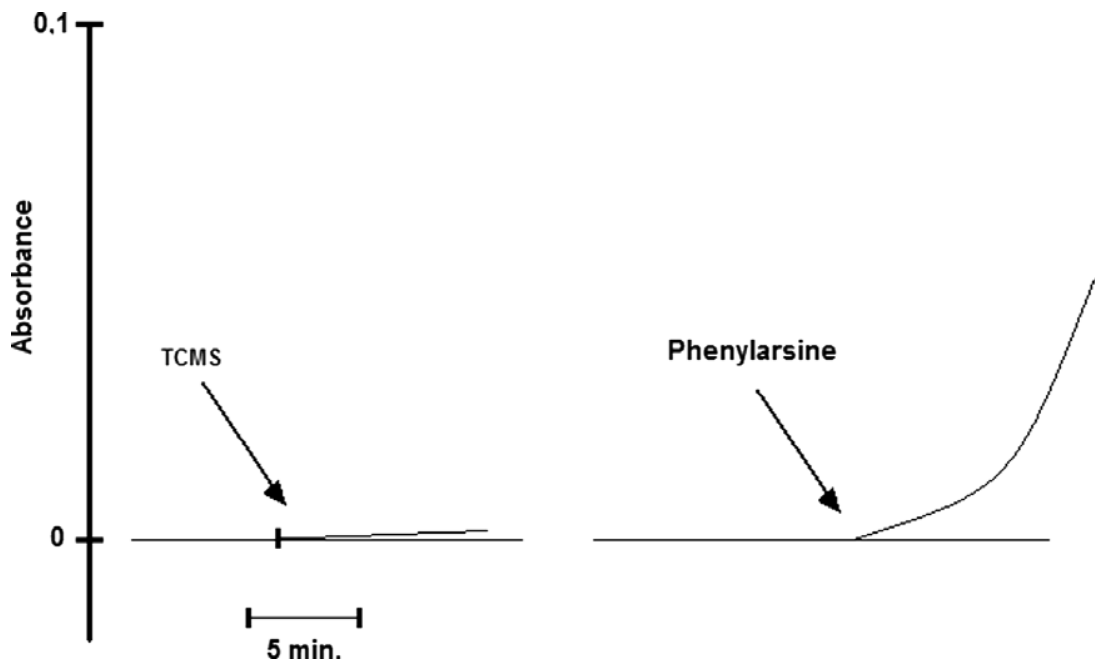

Fig. 6. TCMS does not induce the opening of an MPT pore. Following the addition of the mitochondria (final concentration $0.5 \mathrm{mg} / \mathrm{ml}$ ) to the resuspending medium, the spectrophotometer was adjusted at zero absorbance, as described in 2 . The successive additions of TCMS up to a concentration of $0.4 \mathrm{mM}$, did not induce an absorbance decrease (a) (swelling). On the contrary, in the same conditions, $40 \mu \mathrm{M}$ Phenylarsine which is a typical inductor of the opening of the MPT pore, induces a fast absorbance decrease. 
colloid-osmotic process (Bernardi, 1999). This swelling is evidenced by monitoring the absorbance quenching at $540 \mathrm{~nm}$.

In some cases, the opening of pores for small size solutes (Ichas and Mazat, 1998) is not accompanied by mitochondrial swelling, since the small size of the pores only allows the passage of protons.

In both cases, the opening of a pore causes inhibition of the ATP synthesis, and is inhibited by cyclosporine.

Fig. 6 shows that TCMS does not induce the opening of a large size pores, since no swelling occurs (for comparison, the effect produced by phenylarsine, which is a typical inductor of the opening of the MPT pore, is shown). The opening of small size pores, as a mode of TCMS action can be excluded, since the ATP synthesis (Fig. 2) inhibition is not altered by means of cyclosporine.

\section{Conclusions}

Steps involved in the ATP synthesis were analysed in order to define the mechanism of the inhibitory effect of TCMS on this process.

The analysis of each step allowed us to conclude that TCMS does not act as inhibitor of ATPase (Fig. 4), or uncoupler or detergent (Fig. 5) or inductor of opening of a high size pore (Fig. 6).

It can also be ruled out that the ATP synthesis inhibition is due to opening of a small size pore, since the inhibition is not cyclosporine-sensitive (Fig. 2).

It can be concluded that the TCMS-induced inhibition of the ATP synthesis is likely consequent to RC inhibition. This conclusion is further supported by the substantial overlap of Fig. 2 with that of Fig. 3, which shows the respiratory rate inhibition. Taking into account the structure of the RC (Fig. 1), its inhibition by TCMS when succinate but not glutamate/malate is the substrate, this suggests that the succinic dehydrogenase is the target of TCMS.

Since other reports indicate that TCMS demonstrates environmental characteristics similar to TBT (Voulvoulis et al., 2001), a comparison between this and other antifouling compounds is necessary. There are two crucial points to consider:

- The $\mathrm{LC}_{50}$ values (i.e. the concentration of toxic compound necessary to inhibit $50 \%$ of ATP synthesis in mitochondria).

- The action mechanism.

With regard to the first point, the available literature data refer only to TBT, Znpyrithione, Sea-Nine and Irgarol (Aldridge and Street, 1964; Bragadin et al., 2003, 2005, 2006).

The $\mathrm{LC}_{50}$ value of $0.6 \mu \mathrm{M}$ was found for TBT (Aldridge and Street, 1964), whereas for Irgarol (Bragadin et al., 2006), Znpyrithione (Bragadin et al., 2003), and Sea-Nine (Bragadin et al., 2005), a value of 80,24 and $6 \mu \mathrm{M}$ were found, respectively. Therefore, the "in vitro" mitochondrial toxicity is in the following order:

TBT $>$ Sea-Nine $>$ Znpyrithione $>$ TCMS $>$ Irgarol.

The action mechanism, is also an important point to consider, since the molecular target suggests a similar sensitivity towards the toxic compounds in many organisms. As the $\mathrm{RC}$ is similar in all living organisms, any effect (inhibition) of the same toxic compound on the RC should be similar in many living organisms (presuming that the mitochondria are the preferential target, as it occurs in many cases).

This conclusion can be proposed for both TCMS and Sea-Nine, since, as reported in the literature (Bragadin et al., 2005), the RC is the action site for both compounds.

Furthermore, a comparison between the two antifouling compounds, TCMS and Sea-Nine suggests that, in those living organisms, where the mitochondria are the preferential target, the sensitivity to the antifouling compounds should follow the toxicity order indicated above:

\section{Sea-Nine $>$ TCMS}

Obviously, also the bioavailability of the compounds should be taken into account.

A comparison with other antifouling compounds is not possible, because of the different action mechanism: Irgarol and Znpyrithione cause the opening of a transmembrane pore (Bragadin et al., 2003, 2006). This mechanism is very complicated and not yet completely clarified. Analogously, various action mechanisms concerning mitochondria have been proposed in the case of TBT, including the opening of a transmembrane pore, and different membrane transport mechanisms (Aldridge and Street, 1964; Bragadin and Marton, 1997).

\section{References}

Aldridge, W.N., Street, B.W., 1964. Biochemical effects and properties of trialkyltins. Biochem. J. 91, 287-297.

Azzone, G.F., Pozzan, T., Bragadin, M., Miconi, V., 1979. Thermodynamics and kinetics of the $\mathrm{H}^{+}$proton pump in the mitochondrial electron transport. J. Biol. Chem. 254, 10213-10219.

Bernardi, P., 1999. Mitochondrial transport of cations: channels, exchangers, and permeability transitions. Physiol. Rev. 79, 1127-1155.

Bragadin, M., Dell'Antone, P., 1994. A new in vitro toxicology test based on the response to toxic substances in solution of mitochondria from beef heart. Arch. Environ. Toxicol. Chem. 27, 410-414.

Bragadin, M., Marton, D., 1997. A proposal for a new mechanism of interaction of trialkyltin (TAT) compounds with mitochondria. J. Inorg. Biochem. 68, 75-78.

Bragadin, M., Pozzan, T., Azzone, G.F., 1980. Kinetics of the $\mathrm{Ca}^{2+}$ carrier in rat liver mitochondria. Biochemistry 18, 5972-5978.

Bragadin, M., Manente, S., Marton, D., Cima, F., Rigobello, M.P., Bindoli, A., 2003. The interaction of zinc pyrithione with mitochondria and a study of the mechanism of inhibition of ATP synthesis. Appl. Organomet. Chem. 17, 869-874.

Bragadin, M., Pavoni, B., Scutari, G., Manente, S., 2005. An in vitro study of the interaction of Sea-Nine with mitochondria from rat liver. Environ. Toxicol. Chem. 24 (5), 1074-1078. 
Bragadin, M., Cima, F., Ballarin, L., Manente, S., 2006. Irgarol inhibits the synthesis of ATP in mitochondria from rat liver. Chemosphere 65, 1898-1903.

Huh, W.K., Masuji, Y., Tada, J., Arata, J., Kaniwa, M., 2001. Allergic contact dermatitis froma pyridine derivative in polyvinyl chloride leather. Am. J. Contact Dermat. 12 (1), 35-37.

Ichas, F., Mazat, J.-P., 1998. From calcium signaling to cell death: two conformations for the mitochondrial transition pore: switching from low- to high-conductance state. Biochim. Biophys. Acta 1366, 33-50.

Karlsson, J., Eklund, B., 2004. New biocide antifouling paints are toxic. Mar. Pollut. Bull. 49, 456-464.

Kostantinou, I.K., Albanis, T.A., 2004. Worldwide occurrence and effects of antifouling paint booster biocides in the aquatic environment: a review. Environ. Int. 30, 235-248.

Lowry, O.H., Rosenbrough, H.J., Farr, A.L., Randall, R.J., 1951. Protein measurement with the folin phenol reagent. J. Biol. Chem. 193, 265275 .
Mitchell, P., 1969. Keilin's respiratory chain concept and its chemiosmotic consequences. Science 286, 1148-1159.

Nishimura, M., Ito, T., Chance, B., 1962. Studies on bacterial photophosphorylation. III. A sensitive and rapid method for determination of photophosphorylation. Biochim. Biophys. Acta 7 (59), 1977-1982.

Sabev, H.A., Handley, P.S., Robson, G.D., 2004. In situ quantification of biocide efficacy using GFP transformed Aureobasidium pullutans. J. Appl. Microbiol. 97 (6), 1132-1139.

Thomas, K.V., 1998. Determination of Selected antifouling booster biocides by high-performance liquid chromatography-atmospheric pressure chemical ionization mass spectroscopy. J. Chromatogr. A $825,29-35$.

Voulvoulis, N., Scrimshaw, M.D., Lester, J.N., 2001. Partitioning of selected antifouling in the aquatic environment. Mar. Environ. Res. 53, $1-16$. 